\title{
Selection for Cognitive Control: A Functional Magnetic Resonance Imaging Study on the Selection of Task-Relevant Information
}

\author{
Marcel Brass and D. Yves von Cramon \\ Max Planck Institute for Human Cognitive and Brain Sciences, Department of Cognitive Neurology, 04103 Leipzig, Germany
}

The complex environment we live in makes it necessary to distinguish relevant from irrelevant information constantly and reliably. The aim of the present study was to investigate the neural substrate underlying the selection of task-relevant information. We devised a new paradigm in which participants had to switch between two different tasks that were instructed by task cues. The task cues had a relevant and an irrelevant cue dimension. In congruent trials, both cue dimensions indicated the same task; in incongruent trials, they indicated different tasks; and in neutral trials, only the relevant dimension indicated a task. By comparing trials in which both cue dimensions were informative (congruent and incongruent trials) with trials in which only the relevant dimension was informative (neutral trials), we were able to show that the lateral prefrontal cortex and a region in the intraparietal sulcus are involved in the selection of task-relevant information. Furthermore, the present paradigm allows the influence of the selected task and stimulus dimension to be investigated. No significant influence was found in the prefrontal cortex, indicating that this region serves a very abstract role in the selection of taskrelevant information.

Key words: cognitive control; task selection; fMRI; task switching; prefrontal cortex; intraparietal sulcus

\section{Introduction}

The human ability to selectively adapt behavior to different situations and tasks is a crucial requirement in our daily life (Norman and Shallice, 1986; Monsell, 1996). Our environment generally affords a variety of different behavioral options, only some of which are adaptive in any given situation. Therefore, individuals are required to select the contextual information that determines adaptive, appropriate behavior. We refer to this control process as the selection of task-relevant information.

One crucial question addressed by research on cognitive control concerns the neural mechanisms underlying this selection process. Single-unit recordings in monkeys (Asaad et al., 2000; Wallis et al., 2001; Wallis and Miller, 2003) and neuropsychological studies (Milner, 1963) have demonstrated that the lateral prefrontal cortex plays a crucial role in this respect.

In cognitive psychology, the paradigm most widely used to investigate the selection of contextual information is the Stroop task (MacLeod, 1991). In the Stroop task, participants must identify the color of the ink in which the name of a color is written while suppressing the more automatic response of reading the color name. Neuroimaging data suggest that the frontolateral and frontomedian cortex are involved in this selection process

Received March 1, 2004; revised Aug. 19, 2004; accepted Aug. 20, 2004. We thank Franziska Korb for programming the experiment and performing the testing.

Correspondence should be addressed to Dr. Marcel Brass, Max Planck Institute for Human Cognitive and Brain Sciences, Stephanstrasse 1A, D-04103 Leipzig, Germany. E-mail: brass@cns.mpg.de. DOI:10.1523/JNEUROSCI.2513-04.2004

Copyright $\odot 2004$ Society for Neuroscience $\quad$ 0270-6474/04/248847-06\$15.00/0
(Banich et al., 2000; Milham et al., 2001; Zysset et al., 2001). However, the Stroop task confounds the selection of taskrelevant information with the selection of the appropriate response, because the stimuli indicate both the task and the response.

One way to overcome this shortcoming is to separate the selection of task-relevant information by presenting a task cue before the task, as is commonly done in the context of task switching (Sudevan and Taylor, 1987; Meiran, 1996; Monsell, 2003). We devised a new paradigm in which participants were required to alternate between two simple tasks. A task cue preceding the task indicated which task was to be executed. Like the Stroop task, the task cue had two different dimensions. The relevant dimension could indicate task A or task B. The irrelevant dimension could indicate the same task as the relevant dimension (congruent condition), a different task (incongruent condition), or no task at all (neutral condition). The relevant cue dimension changed between blocks. In this paradigm, selection requirements are manipulated on the task level only.

By comparing the bivalent conditions, in which both dimensions contain task-relevant contextual information (congruent and incongruent), with the univalent condition, in which only one dimension contains task-relevant contextual information (neutral), it is possible to identify the brain regions involved in the selection of the relevant task context. This selection process should be independent of whether the irrelevant stimulus dimension indicates the same or a different task. The contrast of the congruent and incongruent condition, on the other hand, should reveal the areas involved in the selection of a specific task. 


\section{Materials and Methods}

Participants. Eighteen healthy volunteers were recruited. We obtained written consent from all participants before the scanning session. All participants had normal or corrected-to-normal vision. No volunteer had a history of neurological, major medical, or psychiatric disorder. Two participants' data were excluded from the analysis. One participant showed an unusual morphology in the structural magnetic resonance (MR) image; the other was excluded because of technical problems during the scanning. The remaining 16 participants were seven males and nine females (age: mean, 25.0; SD, 2.96) who were all right-handed as assessed by the Edinburgh Inventory (Oldfield, 1971).

Behavioral task. Digits from 1 to 9 (except 5) were presented on the computer screen. Participants had to execute two tasks: judging whether the digit was smaller than or $>5$ (magnitude task) and judging whether the digit was odd or even (parity task). The task they had to execute was instructed by a task cue, which was presented before the target in each experimental trial. The cue had the dimensions color and form (Fig. 1). Each dimension had three different values, which were associated with the magnitude task, the parity task, or no task, respectively. For the form dimension, a square indicated the magnitude task, and a diamond indicated the parity task. An octagon was not associated with either task. For the color dimension, blue indicated the magnitude task, green the parity task, and gray no task. The relevant cue dimension changed between blocks. The color dimension was relevant in one-half of the blocks, the form dimension in the other half. The relevant cue dimension always conveyed task-relevant contextual information, whereas the irrelevant cue dimension could convey the same task information (congruent), different task information (incongruent), or no task information (neutral). Each block comprised 37 trials and was preceded by an instructional cue indicating the relevant cue dimension. Six blocks in which color was relevant were alternated with six blocks in which form was relevant. Each block began with a warm-up trial. Next, 32 experimental trials and four null events were presented in random order. The number of trials in each block was motivated by general considerations. We

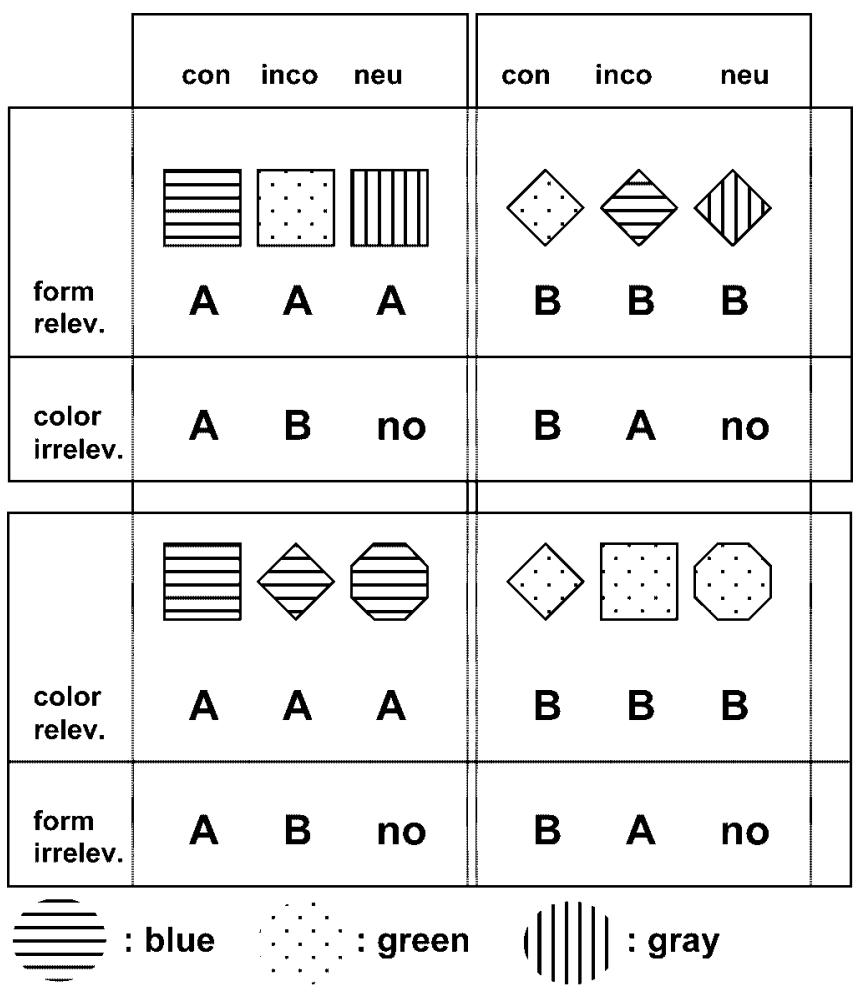

Figure 1. Schematical drawing of the different task cues. Task cues had the dimensions color and form. The relevance of the cue dimension was blocked. Each dimension had three different meanings (task $A$, task $B$, and no task). The combination of the two dimensions resulted in congruent (con), incongruent (inco), and neutral (neu) trials for each relevant dimension. wanted the blocks to be relatively long to give participants the opportunity to adjust to the dimension. At the same time, the blocks should not be too long, because the stimulus dimension of the previous block would otherwise fade out. The randomization was performed over the whole experiment and not for each block separately. In total, the experiment consisted of 384 experimental trials ( 12 blocks of 32 experimental trials) and 48 null events. There were equal proportions of congruent, incongruent, and neutral trials. A brief practice phase of $\sim 10$ min took place outside the scanner before MR scanning began.

Each trial began with a variable jittering interval of 500, 1000, 1500, or $2000 \mathrm{msec}$. A fixation cross was then presented on the screen for 500 msec. In the short cue-target interval (CTI) condition, the fixation cross was followed by a cue presented for $100 \mathrm{msec}$. In the long CTI condition, the cue was presented for $800 \mathrm{msec}$. Participants responded with a left key press to a digit that was smaller than 5 or even and with a right key press to a digit that was $>5$ or odd. The reaction time (RT) window was 2000 msec. After each response, participants were given feedback indicating whether their answer was correct or false. Each trial lasted 6 sec.

Magnetic resonance imaging scanning procedure. The experiment was performed on a 3T scanner (Medspec 30/100; Bruker, Ettlingen, Germany). Twenty axial slices ( $19.2 \mathrm{~cm}$ field of view; $64 \times 64$ matrix; $4 \mathrm{~mm}$ thickness; $1 \mathrm{~mm}$ spacing), parallel to the plane through the anterior and the posterior commissure, and covering the whole brain, were acquired using a single-shot, gradient-recalled echo planar imaging (EPI) sequence (repetition time, $2000 \mathrm{msec}$; echo time, $30 \mathrm{msec}$; $90^{\circ}$ flip angle). Before the functional runs, 20 corresponding anatomical modified driven equilibrium Fourier transformation (MDEFT) slices and 20 structural EPI slices were acquired. Stimuli were presented using a headmounted display with a resolution of $1024 \times 768$ and a refresh rate of 60 $\mathrm{Hz}$.

Functional magnetic resonance imaging analysis. Analysis of functional magnetic resonance imaging (fMRI) data was performed using the inhouse LIPSIA software package (Lohmann et al., 2001). First, functional data were corrected for movement artifacts. The temporal offset between the slices acquired in one scan was then corrected using a sinc interpolation algorithm. Data were filtered using a spatial Gaussian filter with $\sigma=$ 1. A temporal high-pass filter with a cutoff frequency of $1 / 100 \mathrm{~Hz}$ was used for baseline correction of the signal. In addition, a global scaling was performed. All functional data sets were individually registered into three-dimensional (3D) space using the participants' individual highresolution anatomical images. This $3 \mathrm{D}$ reference data set was acquired for each participant during a previous scanning session. The twodimensional anatomical MDEFT slices, geometrically aligned with the functional slices, were used to compute a transformation matrix containing rotational and translational parameters that register the anatomical slices with the $3 \mathrm{D}$ reference $\mathrm{T} 1$ data set. These transformation matrices were normalized to the standard Talairach brain size (Talairach and Tournoux, 1988) by linear scaling and finally applied to the individual functional data. The statistical evaluation was performed using the general linear model for serially autocorrelated observations (Friston et al., 1995). The design matrix for event-related analysis was created using a model of the hemodynamic response with a variable delay. The events for the event-related analysis were set on the cue. Cue and target period were not modeled separately. The model equation was convolved with a Gaussian kernel with a dispersion of $4 \mathrm{sec}$ full width at half maximum. Contrast maps were generated for each participant. Because the individual functional datasets were all aligned to the same stereotactic reference space, a group analysis was then performed. A one-sample $t$ test of contrast maps across participants (random-effects model) was computed to ascertain whether observed differences between conditions were significantly different from zero. Subsequently, $t$ values were transformed into $z$-scores. We only report cortical regions with a $p<0.01(z>2.33$; corrected for multiple comparisons).

To compute the signal change, we determined the most activated voxel of the relevant contrast in the mean $z$-map. From this voxel, we extracted the time course of the signal for each participant from the modeled data. We then subtracted the time course of the null event from the time course of the relevant conditions to attenuate the overlap of the blood oxygenation level-dependent response. We determined the signal change to be 

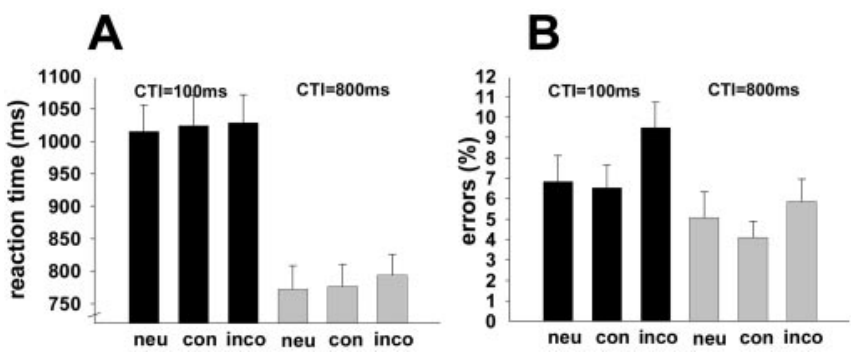

Figure 2. Reaction time $(A)$ and percentage errors $(B)$ as a function of congruency and CTI. con, Congruent; inco, incongruent; neu, neutral. Error bars represent SEM.

the largest value in the time window between 5 and $7 \mathrm{sec}$ after cue presentation.

\section{Results}

\section{Behavioral results}

For the behavioral data we computed an ANOVA with the factors congruency (congruent, incongruent, neutral) and CTI (long, short). There was no significant congruency effect in the RT data $\left(F_{(2,30)}=1.87 ; p=0.17\right)$, but there was a significant main effect for CTI $\left(F_{(1,15)}=227 ; p<0.001\right)$ (Fig. $\left.2 A\right)$. In the error analysis, there was a significant congruency effect $\left(F_{(2,30)}=3.6 ; p<0.05\right)$ and a main effect for CTI $\left(F_{(1,15)}=16.90 ; p<0.01\right)$. No interaction effect was found (Fig. $2 B$ ). Post hoc analysis of the congruency effect in the error data (Fig. $2 B$ ) indicated a significant difference between congruent and incongruent trials $\left(t_{(15)}=3.6\right.$; $p<0.01$ ), a marginally significant difference between incongruent and neutral trials $\left(t_{(15)}=2.1 ; p=0.05\right)$, and no difference between congruent and neutral trials $\left(t_{(15)}=-0.59 ; p=0.55\right)$.

\section{fMRI results}

Because we were primarily interested in the congruency manipulation, and neither the task transition manipulation nor the CTI manipulation yielded a significant activation on the whole-brain level, we pooled the data for these conditions. When comparing both bivalent conditions (congruent and incongruent) with the univalent condition (neutral), an activation was found in the left posterior part of the inferior frontal sulcus (pIFS) (Talairach coordinates: $x=-41, y=18, z=26$ ). This activation was located $\sim 10-15 \mathrm{~mm}$ anterior to the inferior frontal junction area (Brass and von Cramon, 2002, 2004). Furthermore, the ascending branch of the left intraparietal sulcus (IPS) (Talairach coordinates: $x=-37, y=-53, z=47$ ) (Fig. 3) was activated. To test whether areas in the frontomedian cortex might be activated given a more liberal significance criterion, we used an uncorrected $p$-value of $p<0.001$. Given this uncorrected $p$-value, an activation was found in the anterior presupplementary motor area at the border to BA8 $(z=3.8$; Talairach coordinates: $x=7$, $y=18, z=47)$. No significant effect was found for the contrast of congruent and incongruent trials. The signal change analysis (Fig. 3 ) indicated that the pIFS showed a similar level of activation in the congruent and incongruent condition $\left(t_{(15)}=0.2 ; p=\right.$ 0.79 ) but was less activated in the neutral condition (congruent vs neutral: $t_{(15)}=3.6, p<0.01$; incongruent vs neutral: $t_{(15)}=3.9$, $p<0.01)$. There was a numerical difference between the congruent and incongruent condition in the IPS, but it was not significant $\left(t_{(15)}=1.5 ; p=0.16\right)$. Similar to the pIFS, there was a significant difference between the incongruent condition and the neutral condition $\left(t_{(15)}=3.8 ; p<0.01\right)$ and between the congruent condition and the neutral condition $\left(t_{(15)}=3.0 ; p<0.01\right)$. When splitting the conditions for the two different tasks (Fig.
$4 A)$, the pIFS showed no significant main effect for task $\left(F_{(1,15)}<\right.$ $1 ; p=0.55)$ and no interaction with congruency $\left(F_{(2,30)}<1 ; p=\right.$ $0.49)$. There was no difference between the two tasks in the IPS $\left(F_{(1,15)}=2.1 ; p=0.16\right)$ and a marginally significant interaction of congruency and task $\left(F_{(2,30)}=2.9 ; p=0.07\right)$. Furthermore, we investigated the relevant stimulus dimension in the signal change analysis (Fig. $4 B$ ). There was no significant effect of stimulus dimension in the pIFS $\left(F_{(1,15)}<1 ; p=0.68\right)$ and no interaction with congruency $\left(F_{(2,30)}<1 ; p=0.94\right)$. The same held for the IPS. There was no significant difference for stimulus dimension $\left(F_{(1,15)}<1 ; p=0.62\right)$ and no interaction with congruency $\left(F_{(2,30)}=\right.$ $1.3 ; p=0.27)$.

To investigate the influence of practice on the valence effect, we compared the signal change difference for bivalent and univalent trials in the first five trials of each block with the signal change difference in the last five trials of each block (Fig. 5). A small but significant decrease in the valence effect was found in the $\operatorname{pIFS}\left(t_{(15)}=2.49 ; p<0.05\right)$ and a marginally significant effect in the IPS $\left(t_{(15)}=1.98 ; p=0.06\right)$.

\section{Discussion}

The goal of the present study was to investigate the neural correlates underlying the selection of task-relevant information. Because the task cue did not always provide unequivocal information about the relevant task, participants were required to selectively attend to the relevant information in the bivalent conditions (congruent and incongruent). When contrasting these two conditions with the univalent condition (neutral), we found activation in the left lateral prefrontal cortex and the left intraparietal sulcus. Because this selection process was restricted to the processing of the cue, our results demonstrate that in the present paradigm, the pIFS and the IPS are related to the selection of task-relevant information rather than to the selection of the appropriate response.

\section{Selection for cognitive control}

There has been a lengthy debate on the role of the lateral prefrontal cortex in executive control. Although this debate clearly lacks anatomical specificity, there does seem to be some convergence of concepts. In the context of task switching, the left lateral prefrontal cortex has been attributed a general role in task-set representation and response preparation (Braver et al., 2003). Based on studies using the Stroop task, it is assumed that a region in the lateral prefrontal cortex, usually referred to as the dorsolateral prefrontal cortex (DLPFC), is involved in imposing an attentional set (Banich et al., 2000) or implementing cognitive control (MacDonald et al., 2000). In a series of fMRI experiments, the functional role of the lateral prefrontal cortex in Stroop interference was systematically investigated (Banich et al., 2000; Milham et al., 2001, 2002, 2003). Similar to the present experiment, Milham and colleagues (2002) showed that a region in the DLPFC was activated whenever both the relevant and the irrelevant dimension carried task information compared with a condition in which only the relevant dimension carried task information. Furthermore, Milham and colleagues (2001) were able to show that the DLPFC showed an activation regardless of whether the irrelevant dimension was related to the response set. They concluded that the left IFS is involved in imposing an attentional set on the nonresponse level. However, although they managed to disentangle response-related and nonresponse-related processes on the irrelevant stimulus dimension, the Stroop task still confounds these processes on the relevant dimension. Selecting taskrelevant information always confounds response selection. In 
our experiment, we replicated the findings of Milham and colleagues with a design in which the selection of task-relevant information precedes response selection.

Besides overcoming this confounding factor, we were able to investigate whether the task to be selected or the relevant stimulus dimension has an influence on the prefrontal activation. Neither source had a significant effect. This finding indicates that the pIFS serves a very abstract role in the selection of the task context and that it is insensitive to perceptual features of the selected information. An alternative interpretation might be that the pIFS is involved in the inhibition of task-irrelevant information rather than the selection of task-relevant information.

Furthermore, we were also able to demonstrate that the valence effect (incongruent-congruent vs neutral) was influenced by practice. In the first few trials of each block, the previously relevant dimension had a stronger influence on the valence effect in the pIFS than in the last few trials of each block. This supports the assumption that the association between cue dimension and task is relatively weak and fades out when the dimension is no longer relevant.

\section{The contrast of congruent and incongruent trials}

At first glance, it is surprising that the contrast of congruent and incongruent trials did not yield a significant activation, because such a contrast would indicate conflict on the task-selection level. In the Stroop task, a number of prefrontal regions, including the frontolateral cortex and the anterior cingulate cortex, were found to be activated when contrasting congruent and incongruent trials (Banich et al., 2000; Zysset et al., 2001). We assume two main reasons for this difference between the Stroop task and our paradigm. First, in the Stroop task, the irrelevant stimulus dimension is highly overlearned. Therefore, it is very likely that participants are not able to completely suppress the irrelevant cue information. In contrast, in the present design, both dimensions are only weakly associated with the relevant task. Hence, it would seem that in our design, unlike the Stroop task, participants are able to completely suppress the irrelevant cue dimension.

Furthermore, in the Stroop task, unlike our design, the irrelevant stimulus dimension is associated with a specific response, inducing response conflict. This might explain the failure to reveal anterior cingulate activation in our study.

Finally, the failure to identify significant pIFS activation in the contrast of incongruent and congruent trials indicates that the pIFS is related to determine the relevant task selection route rather than task selection per se.

\section{Visual attention and the selection of task-relevant contextual information}

There is an extensive literature investigating attentional selection in the context of visual attention (Corbetta et al., 2000; Pollmann et al., 2000; Corbetta and Shulman, 2002; Liu et al., 2003). These studies have described cortical activation related to spatial (Corbetta et al., 2000; Lepsien and Pollmann 2002) and feature-based attention (Pollmann et al. 2000; Liu et al., 2003). Interestingly, these cortical networks diverge from the network described in the context of selecting task-relevant information (Banich et al., 2000; Dove et al., 2000; MacDonald et al., 2000), indicating that the neuronal mechanisms involved in attentional selection per se and attentional selection in the context of task preparation might
pIFS

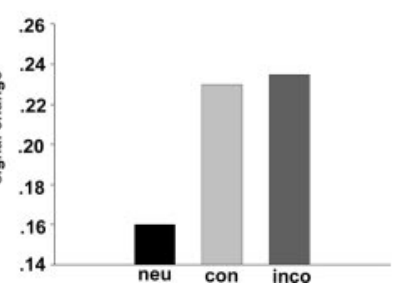

IPS

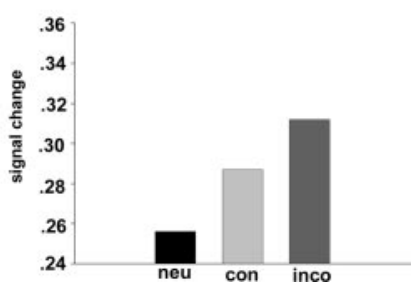

the pIFS and the anterior IPS and signal change as a function of congruency for both regions. con, Congruent; inco, incongruent; neu, neutral.

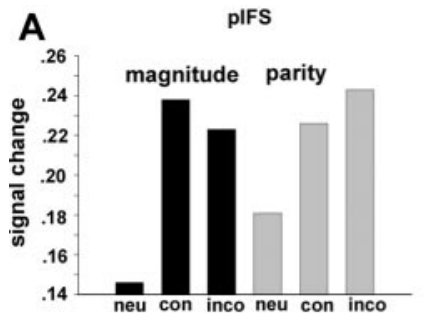

pIFS

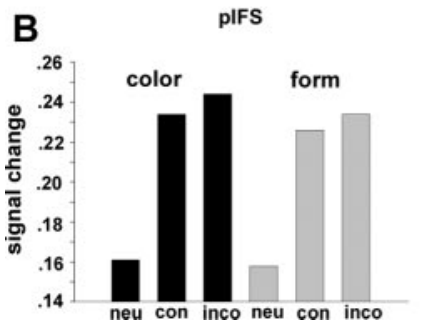

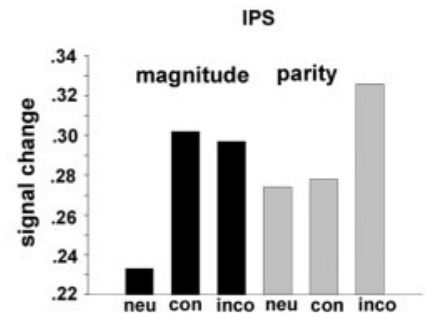

IPS

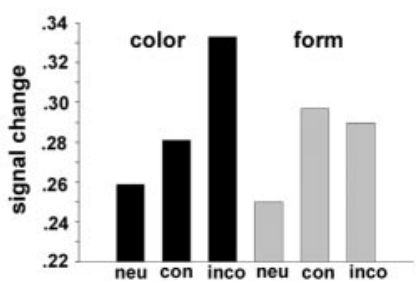

Figure 4. Signal change in the pIFS and IPS as a function of congruency when trials were split for task $(A)$ or relevant stimulus dimension ( $B)$. con, Congruent; inco, incongruent; neu, neutral.
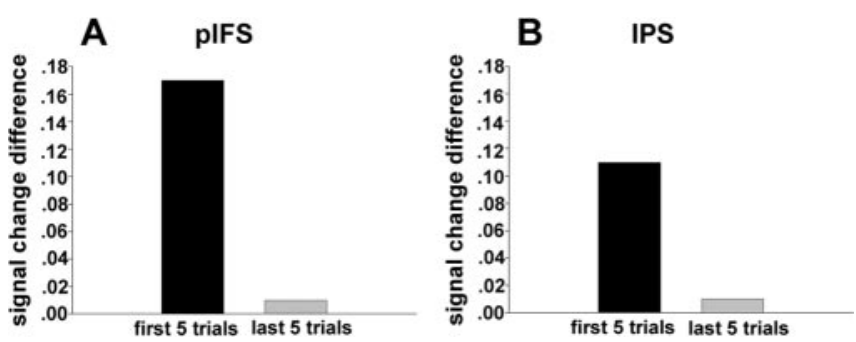

Figure 5. Valence effect (incongruent- congruent vs neutral) for the first five trials of each block compared with the last five trials.

differ (Liu et al., 2003). Recently, Rushworth and colleagues (2001a) argued for such a dissociation by distinguishing between visual switching and response switching. Whereas in the visual switching task, subjects were only required to attend to different visual dimensions, in the response-switching condition, they were required to alternate between different stimulus-response associations. In the present study, participants were always required to select the relevant stimulus dimension. In the neutral condition, the irrelevant stimulus dimension was not related to a task set, although this was the case in the congruent and incongruent conditions. The critical difference between these conditions is not attentional selection per se or selecting the relevant stimulus dimension (Roberts et al., 1988; Rogers et al., 2000), but selecting the information that determines the relevant task context. This would explain why the relevant stimulus dimension had no influence on the valence effect. Similar to the concept of "selection for action," one might distinguish "selection for con- 
trol." We assume that the pIFS serves the function of selecting the relevant task context whenever the information in the environment potentially contains conflicting information.

\section{The posterior IFS and the inferior frontal junction area}

In a number of experiments, we (Brass and von Cramon, 2002, 2004) and others (Konishi et al., 2001; Bunge et al., 2003) have been able to show that a region at the junction of the inferior frontal sulcus and inferior precentral sulcus (IFJ) is involved in the activation of the relevant task representation. This region is located $\sim 10-15 \mathrm{~mm}$ more posterior than the region found in the present experiment. But how are these two regions related to cognitive control? In line with a recent proposal by Koechlin and colleagues (2003), we and others assume a hierarchical organization within the lateral prefrontal cortex (Monchi et al., 2001; Nagahama et al., 2001; Dreher et al., 2002; Forstmann et al., 2004). Although the IFJ directly connects the context information (the cue) to the relevant task representation, the pIFS region makes it possible to selectively attend to specific information and ignore other information. In this sense, the more anterior located region allows us to determine the relevance of environmental information in the context of a broader temporal perspective. In line with this assumption, Monchi and colleagues (2001) assumed that the lateral prefrontal cortex is involved when current information has to be related to previous events stored in working memory. In the terminology of research on working memory, this would mean the manipulation of information in working memory (Owen et al., 1999; Fletcher and Henson, 2001).

\section{The contribution of the parietal cortex}

So far, we have focused our discussion on the frontolateral cortex. However, the left IPS showed an activational pattern similar to the pIFS activation. It has been argued that the IPS is involved in the specification of visuomotor transformation rules or motor attention (Rushworth et al., 2001b). On the other hand, the IPS plays a role in feature-based attention (Corbetta and Shulman, 2002; Liu et al., 2003). The localization of the present IPS activation in the anterior part of the left IPS suggests that it is related to motor attention rather than visual selection (Rushworth et al., 2001b; Corbetta and Shulman, 2002). This interpretation of the IPS activation might help to explain the functional dissociation of the lateral prefrontal cortex and the IPS in cognitive control. As we have suggested previously (Brass and von Cramon, 2004), one interpretation might be that the prefrontal cortex selects an abstract task representation which is then specified in intraparietal areas.

\section{References}

Asaad WF, Rainer G, Miller EK (2000) Task-specific neural activity in the primate prefrontal cortex. J Neurophysiol 84:451-459.

Banich MT, Milham MP, Atchley RA, Cohen NJ, Webb A, Wszalek T, Kramer AF, Liang Z, Barad V, Gullett D, Shah C, Brown C (2000) Prefrontal regions play a predominant role in imposing an attentional 'set': evidence from fMRI. Brain Res Cogn Brain Res 10:1-9.

Brass M, von Cramon DY (2002) The role of the frontal cortex in task preparation. Cereb Cortex 12:908-914.

Brass M, von Cramon DY (2004) Decomposing components of task preparation with functional MRI. J Cogn Neurosci 16:609-620.

Braver TS, Reynolds JR, Donaldson DI (2003) Mechanisms of transient and sustained cognitive control during task switching. Neuron 39:713-726.

Bunge SA, Kahn I, Wallis JD, Miller EK, Wagner AD (2003) Neural circuits subserving the retrieval and maintenance of abstract rules. J Neurophysiol 90:3419-3428.
Corbetta M, Shulman GL (2002) Control of goal-directed and stimulusdriven attention in the brain. Nat Rev Neurosci 3:201-215.

Corbetta M, Kincade JM, Ollinger JM, McAvoy MP, Shulman GL (2000) Voluntary orienting is dissociated from target detection in human posterior parietal cortex. Nat Neurosci 3:292-297.

Dove A, Pollmann S, Schubert T, Wiggins CJ, von Cramon DY (2000) Prefrontal cortex activation in task switching: an event-related fMRI study. Brain Res Cogn Brain Res 9:103-109.

Dreher JC, Koechlin E, Ali SO, Grafman J (2002) The roles of timing and task order during task switching. NeuroImage 17:95-109.

Fletcher PC, Henson RN (2001) Frontal lobes and human memory: insights from functional neuroimaging. Brain 124:849-881.

Forstmann B, Brass M, Koch I, von Cramon DY (2004) Investigating endogeneous control in task switching. Neuropsychologia, in press.

Friston KJ, Holmes AP, Poline JB, Grasby PJ, Williams SC, Frackowiak RS, Turner R (1995) Analysis of fMRI time-series revisited. NeuroImage 2:45-53.

Koechlin E, Ody C, Kouneiher F (2003) The architecture of cognitive control in the human prefrontal cortex. Science 302:1181-1185.

Konishi S, Donaldson DI, Buckner RL (2001) Transient activation during block transition. NeuroImage 13:364-374.

Lepsien J, Pollmann S (2002) Covert reorienting and inhibition of return: an event-related fMRI study. J Cogn Neurosci 14:127-144.

Liu T, Slotnick SD, Serences JT, Yantis S (2003) Cortical mechanisms of feature-based attentional control. Cereb Cortex 13:1334-1343.

Lohmann G, Muller K, Bosch V, Mentzel H, Hessler S, Chen L, Zysset S, von Cramon DY (2001) LIPSIA-a new software system for the evaluation of functional magnetic resonance images of the human brain. Comput Med Imaging Graph 25:449-457.

MacDonald 3rd AW, Cohen JD, Stenger VA, Carter CS (2000) Dissociating the role of the dorsolateral prefrontal and anterior cingulate cortex in cognitive control. Science 288:1835-1838.

MacLeod CM (1991) Half a century of research on the Stroop effect: an integrative review. Psychol Bull 109:163-203.

Meiran N (1996) Reconfiguration of processing mode prior to task performance. J Exp Psychol Learn Mem Cogn 22:1423-1442.

Milham MP, Banich MT, Webb A, Barad V, Cohen NJ, Wszalek T, Kramer AF (2001) The relative involvement of anterior cingulate and prefrontal cortex in attentional control depends on nature of conflict. Brain Res Cogn Brain Res 12:467-473.

Milham MP, Erickson KI, Banich MT, Kramer AF, Webb A, Wszalek T, Cohen NJ (2002) Attentional control in the aging brain: insights from an fMRI study of the Stroop task. Brain Cogn 49:277-296.

Milham MP, Banich MT, Claus ED, Cohen NJ (2003) Practice-related effects demonstrate complementary roles of anterior cingulate and prefrontal cortices in attentional control. NeuroImage 18:483-493.

Milner B (1963) Effects of different brain lesions on card sorting. Arch Neurol 9:90-100.

Monchi O, Petrides M, Petre V, Worsley K, Dagher A (2001) Wisconsin card sorting revisited: distinct neural circuits participating in different stages of the task identified by event-related functional magnetic resonance imaging. J Neurosci 21:7733-7741.

Monsell S (1996) Control of mental processes. In: Mysteries of the mind: tutorial essays on cognition (Bruce V, ed), pp 93-148. Hove, UK: Erlbaum.

Monsell S (2003) Task switching. Trends Cogn Sci 7:134-140.

Nagahama Y, Okada T, Katsumi Y, Hayashi T, Yamauchi H, Oyanagi C, Konishi J, Fukuyama H, Shibasaki H (2001) Dissociable mechanisms of attentional control within the human prefrontal cortex. Cereb Cortex 11:85-92.

Norman DA, Shallice T (1986) Attention to action: willed and automatic control of behavior. In: Consciousness and self-regulation (Davidson RJ, Schwartz GE, Shapiro D, eds), pp 1-18. New York: Plenum.

Oldfield RC (1971) The assessment and analysis of handedness: the Edinburgh inventory. Neuropsychologia 9:97-113.

Owen AM, Herrod NJ, Menon DK, Clark JC, Downey SP, Carpenter TA, Minhas PS, Turkheimer FE, Williams EJ, Robbins TW, Sahakian BJ, Petrides M, Pickard JD (1999) Redefining the functional organization of working memory processes within human lateral prefrontal cortex. Eur J Neurosci 11:567-574. 
Pollmann S, Weidner R, Muller HJ, von Cramon DY (2000) A frontoposterior network involved in visual dimension changes. J Cogn Neurosci 12: $480-494$.

Roberts AC, Robbins TW, Everitt BJ (1988) The effects of intradimensional and extradimensional shifts on visual discrimination learning in humans and nonhuman primates. Q J Exp Psychol 40B:321-341.

Rogers RD, Andrews TC, Grasby PM, Brooks DJ, Robbins TW (2000) Contrasting cortical and subcortical activations produced by attentional-set shifting and reversal learning in humans. J Cogn Neurosci 12:142-162.

Rushworth MF, Krams M, Passingham RE (2001a) The attentional role of the left parietal cortex: the distinct lateralization and localization of motor attention in the human brain. J Cogn Neurosci 13:698-710.
Rushworth MF, Paus T, Sipila PK (2001b) Attention systems and the organization of the human parietal cortex. J Neurosci 21:5262-5271.

Sudevan P, Taylor DA (1987) The cuing and priming of cognitive operations. J Exp Psychol Hum Percept Perform 13:89-103.

Talairach J, Tournoux P (1988) Co-planar stereotaxic atlas of the human brain. Stuttgart, Germany: Georg Thieme Verlag.

Wallis JD, Miller EK (2003) From rule to response: neuronal processes in the premotor and prefrontal cortex. J Neurophysiol 90:1790-1806.

Wallis JD, Anderson KC, Miller EK (2001) Single neurons in prefrontal cortex encode abstract rules. Nature 411:953-956.

Zysset S, Muller K, Lohmann G, von Cramon DY (2001) Color-word matching Stroop task: separating interference and response conflict. NeuroImage 13:29-36. 